\title{
Medicinal plants and practices of Rongkong Traditional Healers in South Sulawesi, Indonesia
}

\author{
FANIE INDRIAN MUSTOFA ${ }^{1, \boldsymbol{v}}$, NUNING RAHMAWATI ${ }^{1}$, AMINULLAH $^{2, v \varphi}$ \\ ${ }^{1}$ Medicinal Plant and Traditional Medicine Research and Development Center, National Institute Health Research and Development, Ministry of Health, \\ J1. Raya Lawu, Tawangmangu, Karanganyar 57792, Central Java, Indonesia. Tel./fax.: +62-271-697010, "email: fanie.mustofa@ gmail.com \\ ${ }^{2}$ Faculty of Pharmacy, Universitas Hasanuddin. Jl. Perintis Kemerdekaan Km. 10, Tamalanrea, Makassar 90245, South Sulawesi, Indonesia. \\ Tel./fax.: +62-411-588566, ^vemail: aminullahygeia@yahoo.co.id
}

Manuscript received: 1 October 2019. Revision accepted: 20 January 2020.

\begin{abstract}
Mustofa FI, Rahmawati N, Aminullah. 2020. Medicinal plants and practices of Rongkong Traditional Healers in South Sulawesi, Indonesia. Biodiversitas 21: 642-651. Ethnomedicine reflects the fusion of tradition, natural resources, and local knowledge of the community in maintaining health. However, this knowledge is facing the threat of disappearance due to poor documentation and socio-cultural alteration. This study was conducted to find out information about species of plants used and related practices by traditional healers to overcome health problems. The study was conducted in Rongkong, North Luwu District, South Sulawesi Province. A semi-structured questionnaire was developed to obtain informants demographic data, species of medicinal plants, traditional medicine ingredients, parts used, methods of remedies preparation and use, as well as various ailments that can be treated by medicinal plants. The data were analyzed descriptively and quantitatively, by calculating the family and species use-value. The study revealed medicinal plants used and practices by Rongkong traditional healers to treat 31 ailments. They used a total of 62 species of medicinal plants distributed among 33 botanical families and 55 genera. Allium cepa has the highest UV among all medicinal plants in Rongkong. Both traditional knowledge and medicinal plant diversity possessed by Rongkong community are valuable local assets that must be maintained and developed for greater benefits.
\end{abstract}

Keywords: Medicinal plant, North Luwu, Rongkong, traditional healer

\section{INTRODUCTION}

The use of medicinal plants in Indonesia has been known by generations, and has empirically proven to overcome community health problems for years. Indonesia has hundreds of ethnic communities that possessed various local knowledge related to traditional medicine. The knowledge was transferred orally from traditional healers, ethnic leaders, or ordinary people to their students as well as their offspring. That practice has the risk of losing crucial knowledge since there is almost no type of documentation of it. Acculturation and modernization are not actually the main threats to traditional medicinal practices, but they should ideally support the dynamic character of traditional medicine knowledge (Weckerle et al. 2018). However, forest conversion, environmental damage and continuous harvest from the wild lead to the endangerment and extinction of medicinal plants which are the main ingredients of traditional medicines (Giday et al. 2010; Silalahi 2018).

In many communities, traditional healers have important role in local health system. They uphold tradition by getting along with nature to maintain the community's health (Cheikhyoussef et al. 2011). People who live in remote areas and have limited access to formal health services are more dependent on traditional healers (Suharmiati et al. 2012; Dermawan 2013). In rural areas, people are also still dependent on the forests for the supply of their needs, such as fuelwood, timber, and medicine
(Amri and Kisangau 2012). Native people living in their own region commonly rely on the surrounding nature and possess local wisdom regarding using medicinal plants for healthcare (Ghimire et al. 2018).

Rongkong is one of the indigenous ethnic groups in South Sulawesi, Indonesia. This community has specific local knowledge in using natural resources for various purposes, including medical purposes. They live in isolated areas which are far and difficult to access, and are also surrounded by mountains that block the entry of communication networks. The formal health facilities consist only of a Public Health Center and two Public Health Sub-centers. However, these facilities are not enough to meet the needs of the large population and the wide area of this sub-district.

Rongkong traditional healers have played an important role in maintaining community health in the background of limited access to formal health facilities. Rongkong highland is surrounded by mountains with fertile soil supporting the growth of plant diversity, which provides sufficient raw materials as healing ingredients.

Previous studies related to traditional knowledge of Rongkong community restricted to ethnobotanical documentation of plants in a single village called Rinding Allo for various purposes, such as food, medicine, building, traditional rituals, and handicrafts. The study revealed the use of 28 species of medicinal plants (Wartika et al. 2015). Plants used specifically to manage health problems by traditional healers of Rongkong have not been studied so 
far. The present study was, therefore, undertaken with the above-mentioned objective.

\section{MATERIALS AND METHODS}

\section{Study area}

The study was conducted in April 2017 at Rongkong Sub-district, North Luwu District, South Sulawesi, Indonesia. It is located at south latitude of $20^{\circ} 24^{\prime} 23^{\prime \prime}$ $20^{\circ} 40^{\prime} 31^{\prime \prime}$ and the east longitude of $119^{\circ} 47^{\prime} 46^{\prime \prime}-120^{\circ} 5^{\prime} 36^{\prime \prime}$. The total area is $771.50 \mathrm{~km}^{2}$ and the altitude reaches 1107 $1502 \mathrm{~m}$ above sea level. The earlier name of this subdistrict was Limbong, which means puddle in local language. In 2015, the sub-district name was changed to Rongkong, which came from local words ma'rongko which means luck. Rongkong is located in a valley surrounded by mountains, the administration boundary is North Toraja District at the west side, Sabbang Sub-district at the east and south side, and Seko Sub-district at the north side. Rongkong Sub-district has seven villages, but the present study was conducted only in four villages: Limbong, Marampa, Rinding Allo and Komba (Figure 1).

\section{Methodology}

This study involved five well known traditional healers who possessed knowledge and expertise in healing and treating diseases by using medicinal plants. The informants were chosen by purposive sampling method based on information given by community leaders, tribal heads, village heads, informal leaders, health offices, health centers, and other reliable sources.

Data collection was carried out by interviews, observations, and retrieval of medicinal plant specimens. A semi-structured questionnaire was developed to obtain informants demographic data, species of medicinal plants used, parts used, methods of traditional medicine preparation and use, as well as health problems treated. Open interviews were also conducted to obtain different concepts of traditional medicine from the traditional healer. Then an overview of the ecological conditions of the traditional healer's dwelling place and the habitats of medicinal plants was obtained by observations. Plant specimens were collected from natural habitats for identification purposes. Botanical identity of voucher specimens was confirmed with the help of experts from Gadjah Mada University, Sebelas Maret University, Andalas University, Mulawarman University, and Research Centre for Biology, Indonesian Institute of Sciences. All of the scientific names have been checked with www.theplantlist.org. The voucher specimens are deposited in the form of photos and herbaria at Herbarium Tawangmanguensis, Karanganyar, Central Java.

\section{Data analysis}

Data about local knowledge and medicinal plant species used to treat health problems were analyzed descriptively. Family Use Value (FUV) to find out the relative importance of families to distinguish plant families that have more uses than would be expected by random chance was s calculated as follows (Hoffman and Gallaher 2007):

$$
\mathrm{FUV}=\sum \mathrm{UV}_{\mathrm{s}} / \mathrm{n}_{\mathrm{s}}
$$

FUV was calculated by summing the use-values for all the species within a given family and dividing it by total number of species within a given family $\left(\mathrm{n}_{\mathrm{s}}\right)$. Use Value (UV) which is the relative importance of a species used locally was calculated as follows (Gazzaneo et al. 2005; Alzweiri et al. 2011):

$$
\mathrm{UV}_{\mathrm{s}}=\sum \mathrm{Ui} / \mathrm{n}
$$

$\mathrm{Ui}$ is the number of use-reports cited by each informant for a given species and $\mathrm{n}$ refers to the total number of informants. Use values are high when there are many usereports for a plant.

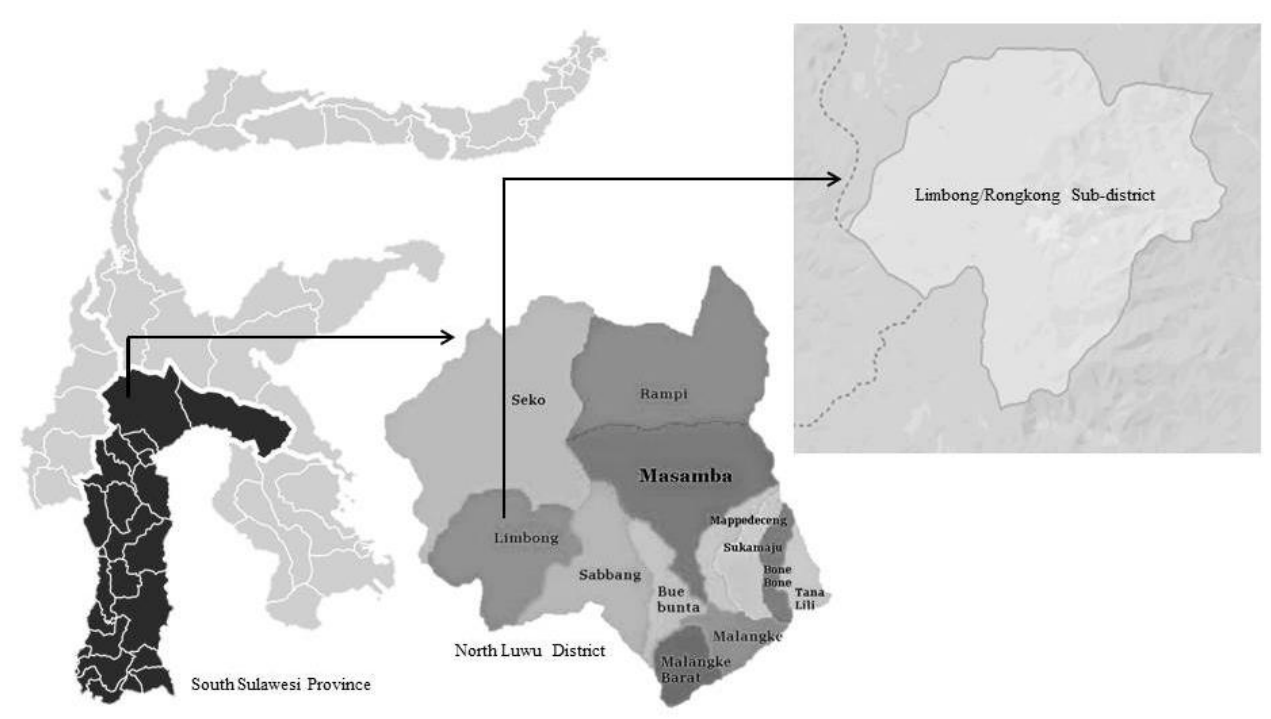

Figure 1. Location of Limbong/Rongkong Sub-district, North Luwu District, South Sulawesi Province 
Table 1. Informants Demography

\begin{tabular}{cccccc}
\hline No & Sex & Age (Year) & Education & Occupation & Villages \\
\hline 1 & Male & 73 & Elementary School & Farmer & Borong Lewa, Limbong \\
2 & Female & 45 & High School & Farmer & Borong Lewa, Limbong \\
3 & Female & 60 & Elementary School & Farmer & Ponglegen, Marampa \\
4 & Female & 73 & Elementary School & Farmer & Manganan, Rinding Allo \\
5 & Female & 64 & Elementary School & Farmer & Komba, Komba \\
\hline
\end{tabular}

\section{RESULTS AND DISCUSSION}

\section{Results}

Information on medicinal plants used to treat various ailments was obtained from five traditional healers. All informants are indigenous inhabitants of Rongkong, and settled in this area from their childhood. The brief profile of the informants is given in Table 1.

A total of 112 medicinal plant uses that represent 67 formulas were collected from the study area. They used 62 species of medicinal plants distributed among 33 botanical families and 55 genera (Table 2). Vernacular names indicate local or trade names that have popular in Indonesia. The recommended uses of medicinal plants for different health problems, parts used, route of administration (RoA) as well as composition of each remedy is provided in the table.

Out of the 62 species, 22 species are used to treat more than one ailment. Each of the remaining 40 species used to treat only one ailment each. A.cepa has the highest UV (2.2), followed by A.conyzoides, H.capitata, and Z.officinale (1.0). Other species have UV values ranging between 0.2 to 0.8 . Table 2 shows various routes of administration (RoA), such as oral, topical, ear drop, eye drop, blown hair wash, mouthwash, and bath. There are two types of herbal remedies by composition, single and a mixture of two or more plant parts.

Figure 2 reveals the names of 11 families that have at least two species of medicinal plants each. A total of 41 species or more than $50 \%$ belong to 11 families (Fig.2). The remaining 22 families consist of one species each. The largest number of species is from Poaceae (7 of 62 species), followed by Asteraceae (six of 62 species), and Lamiaceae and Zingiberaceae (five of 62 species, each). The other families have two to four species, each.

Figure 3 shows 14 families high Family Use Value (FUV), with at least two citations per family. The highest FUV was for Amaryllidaceae (1.20). Both Convolvulaceae and Piperaceae were in the second position, both with the same FUV (0.60). The remaining 18 families have only one citation among the 112 remedies reported in the present study.

As shown in Figure 4, the informants used various parts of the plants for preparing the remedies, such as leaf, rhizome, tuber, whole plant, fruit, stem, seed, root, exudate, shoot, and water. Leaf was the most abundant part used by informants. More than half $(52.68 \%)$ of all remedies reported used leaf as the main material. Its followed by the use of rhizome $(11.61 \%)$, tuber $(9.82 \%)$ and whole plant $(8.93 \%)$ Whole plant refer to all parts of the herbaceous plant present above the soil. Exudate of Jatropha curcas and water of Cocos nucifera were also used.

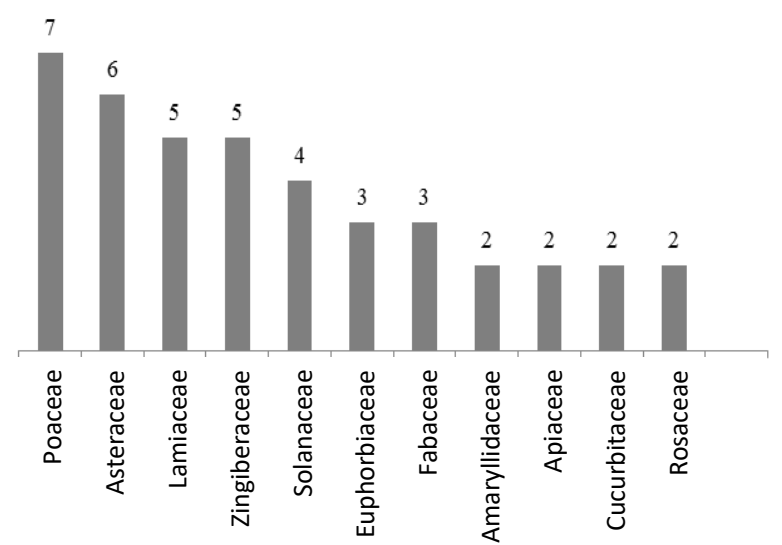

Figure 2. Families with two or more species of medicinal plants

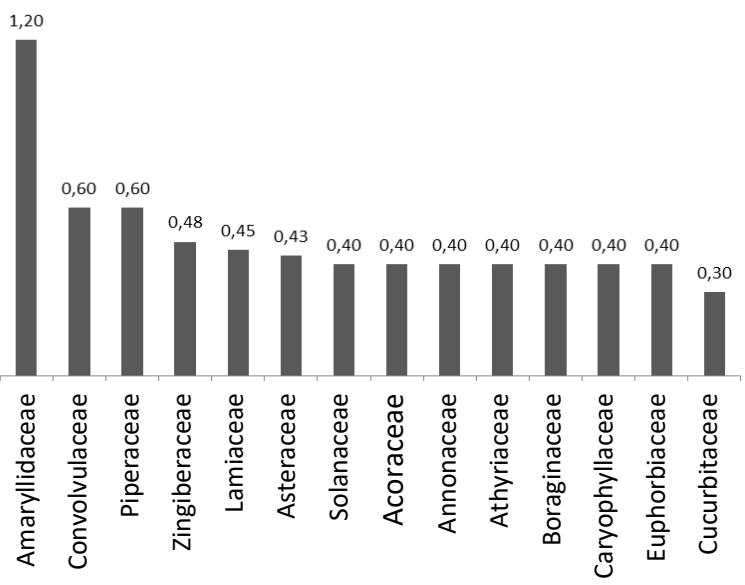

Figure 3. Families with high Family Use Value (FUV)

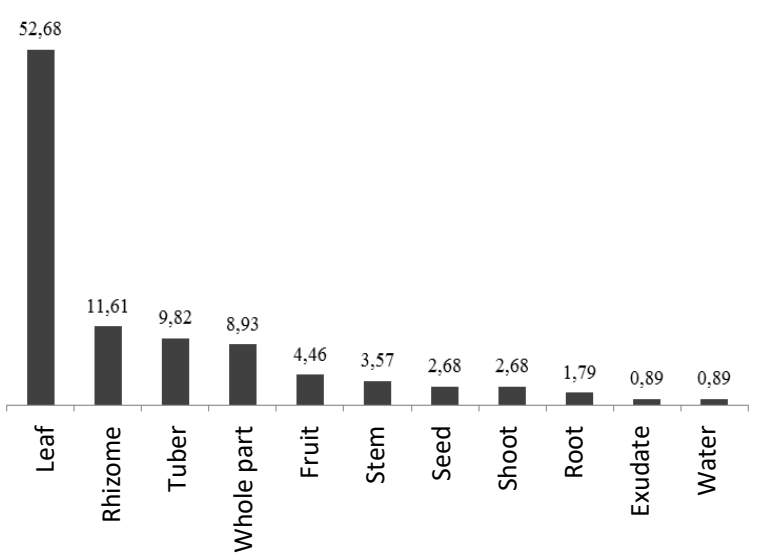

Figure 4. Percentage of medicinal plant parts used by the informants 
Table 2. Medicinal Plants used for treatment of various ailments in Rongkong, South Sulawesi, Indonesia

\begin{tabular}{|c|c|c|c|c|c|c|}
\hline Family & $\begin{array}{l}\text { Scientific name } \\
\text { (Vernacular name) }\end{array}$ & Recommended used & $\mathbf{U v}$ & $\begin{array}{l}\text { Part } \\
\text { used }\end{array}$ & RoA & Compo-sition \\
\hline Acoraceae & $\begin{array}{l}\text { Acorus calamus } \mathrm{L} . \\
\text { (kariango/dringo) }\end{array}$ & $\begin{array}{l}\text { Maternity care, } \\
\text { Undefined ailments }\end{array}$ & 0.4 & $\begin{array}{l}\text { Stem, } \\
\text { Rhizome }\end{array}$ & Topical & Single \\
\hline \multirow[t]{2}{*}{ Amaryllidaceae } & $\begin{array}{l}\text { Allium cepa } \mathrm{L} \text {. } \\
\text { (bawang merah) }\end{array}$ & $\begin{array}{l}\text { Smallpox, dental and oral } \\
\text { problem, cough, ulcer, } \\
\text { fever, neonatal care, } \\
\text { undefined ailments }\end{array}$ & 2.2 & Tuber & $\begin{array}{l}\text { Topical, } \\
\text { oral, }\end{array}$ & $\begin{array}{l}\text { Single, } \\
\text { mixture }\end{array}$ \\
\hline & $\begin{array}{l}\text { Allium schoenoprasum } \mathrm{L} \\
\text { (daun ganda/kucai) }\end{array}$ & Ear problem & 0.2 & Leaf & Eardrop & Single \\
\hline Annonaceae & $\begin{array}{l}\text { Annona muricata } \mathrm{L} \text {. } \\
\text { (Sirikaya/Sirsak) }\end{array}$ & $\begin{array}{l}\text { Beauty care/cosmetics, } \\
\text { high blood pressure }\end{array}$ & 0.4 & Leaf & $\begin{array}{l}\text { Oral, hair } \\
\text { wash }\end{array}$ & Single \\
\hline \multirow[t]{2}{*}{ Apiaceae } & $\begin{array}{l}\text { Centella asiatica (L.) Urb. } \\
\text { (Tambaga/pegagan) }\end{array}$ & Gastritis & 0.2 & Leaf & Oral & Mixture \\
\hline & $\begin{array}{l}\text { Eryngium foetidum } \mathrm{L} \text {. } \\
\text { (Sinaduri/walangi) }\end{array}$ & Heart disease & 0.2 & Herbs & Oral & Mixture \\
\hline Araceae & $\begin{array}{l}\text { Colocasia esculenta (L.) Schott } \\
\text { (Dandupe/keladi) }\end{array}$ & Maternity care & 0.2 & Leaf & Oral & Single \\
\hline Arecaceae & Cocos nucifera L. (Kelapa) & High blood pressure & 0.2 & Water & Oral & Mixture \\
\hline \multirow[t]{6}{*}{ Asteraceae } & $\begin{array}{l}\text { Ageratum conyzoides L. (Bosi- } \\
\text { bosi/bandotan) }\end{array}$ & $\begin{array}{l}\text { Gastritis, magical and } \\
\text { spiritual problem, open } \\
\text { wound }\end{array}$ & 1.0 & Leaf & Oral, topical & $\begin{array}{l}\text { Single, } \\
\text { mixture }\end{array}$ \\
\hline & $\begin{array}{l}\text { Artemisia vulgaris L. (Rumput } \\
\text { cina/baru cina) }\end{array}$ & Gastritis, anti-toxic & 0.4 & Herba & Oral & $\begin{array}{l}\text { Mixture, } \\
\text { single }\end{array}$ \\
\hline & Bidens pilosa L. (Teda/ketul) & $\begin{array}{l}\text { Magical and spiritual } \\
\text { problem }\end{array}$ & 0.2 & Leaf & Topical & \\
\hline & $\begin{array}{l}\text { Crassocephalum crepidioides } \\
\text { (Benth.) S.Moore (Nippon/sintrong) }\end{array}$ & Open wound & 0.6 & Leaf & Topical & $\begin{array}{l}\text { Single, } \\
\text { mixture }\end{array}$ \\
\hline & $\begin{array}{l}\text { Pseudoelephantopus spicatus (Bl.) } \\
\text { C.F. Baker (unknown local name) }\end{array}$ & Appendicitis & 0.2 & Leaf & Oral & Single \\
\hline & $\begin{array}{l}\text { Vernonia amygdalina Delile (daun } \\
\text { klorofil/daun afrika) }\end{array}$ & $\begin{array}{l}\text { High cholesterol, high } \\
\text { blood pressure }\end{array}$ & 0.4 & Leaf & Oral & Mixture \\
\hline Athyriaceae & $\begin{array}{l}\text { Diplazium esculentum (Retz.) Sw. } \\
\text { (Pakis) }\end{array}$ & $\begin{array}{l}\text { Eye problem, maternity } \\
\text { care }\end{array}$ & 0.4 & Leaf & $\begin{array}{l}\text { Eye drop, } \\
\text { topical }\end{array}$ & Single \\
\hline Basellaceae & $\begin{array}{l}\text { Anredera cordifolia (Ten.) Steenis } \\
\text { (Binahong) }\end{array}$ & High cholesterol & 0.2 & Leaf & Oral & Mixture \\
\hline Blechnaceae & $\begin{array}{l}\text { Blechnum capense }(\mathrm{L} .) \text { Schltdl. } \\
\text { (Manuk-manuk/paku) }\end{array}$ & Ulcer & 0.2 & Shoot & Topical & Single \\
\hline Boraginaceae & $\begin{array}{l}\text { Symphytum officinale L. (Lapa- } \\
\text { lapa/kompri) }\end{array}$ & $\begin{array}{l}\text { Maternity care, open } \\
\text { wound }\end{array}$ & 0.4 & Leaf & Oral, topical & Single \\
\hline Caryophyllaceae & $\begin{array}{l}\text { Drynaria cordata (L.) Willd. ex } \\
\text { Roem. \& Schult. } \\
\text { (Ruminding/semanggi) }\end{array}$ & $\begin{array}{l}\text { Magical and spiritual } \\
\text { problem, maternity care }\end{array}$ & 0.4 & Herba & Topical & Mixture \\
\hline Commelinaceae & $\begin{array}{l}\text { Commelina benghalensis L. } \\
\text { (Lesoang) }\end{array}$ & $\begin{array}{l}\text { Magical/spiritual } \\
\text { problem }\end{array}$ & 0.2 & Leaf & Topical & Mixture \\
\hline Convolvulaceae & $\begin{array}{l}\text { Ipomoea batatas (L.) Lam. } \\
\text { (Dandoa/ubi jalar) }\end{array}$ & Maternity care & 0.6 & Leaf & Topical & $\begin{array}{l}\text { Mixture, } \\
\text { single }\end{array}$ \\
\hline \multirow[t]{2}{*}{ Cucurbitaceae } & $\begin{array}{l}\text { Momordica charantia } \mathrm{L} \text {. } \\
\text { (Paria/pare) }\end{array}$ & Cough, fever & 0.4 & Leaf & Oral, topical & Mixture \\
\hline & $\begin{array}{l}\text { Sechium edule (Jacq.) Sw. } \\
\text { (Rabbisa/labu siam) }\end{array}$ & High blood pressure & 0.2 & Fruit & Oral & Single \\
\hline Dennstaedtiaceae & $\begin{array}{l}\text { Pteridium aquilinum (L.) Kuhn. } \\
\text { (Paku lando) }\end{array}$ & Breastmilk booster & 0.2 & Shoot & Topical & Single \\
\hline \multirow[t]{3}{*}{ Euphorbiaceae } & $\begin{array}{l}\text { Aleurites moluccanus (L.) Willd. } \\
\text { (Kemiri) }\end{array}$ & Smallpox & 0.2 & Fruit & Oral, topical & Mixture \\
\hline & $\begin{array}{l}\text { Jatropha curcas L. (Pallang/jarak } \\
\text { pagar) }\end{array}$ & $\begin{array}{l}\text { Sore throat, dental and } \\
\text { oral problem, magical } \\
\text { and spiritual problem, } \\
\text { maternity care }\end{array}$ & 0.8 & $\begin{array}{l}\text { Leaf, } \\
\text { Eksudat }\end{array}$ & $\begin{array}{l}\text { Oral, } \\
\text { topical, bath }\end{array}$ & Single \\
\hline & $\begin{array}{l}\text { Manihot esculenta Crantz. } \\
\text { (Singkong) }\end{array}$ & Gastritis & 0.2 & Leaf & Topical & Single \\
\hline Fabaceae & $\begin{array}{l}\text { Arachis hypogaea L. (Kacang } \\
\text { tanah) }\end{array}$ & Breastmilk booster & 0.2 & Seed & Oral & Single \\
\hline
\end{tabular}




\begin{tabular}{|c|c|c|c|c|c|c|}
\hline & $\begin{array}{l}\text { Lablab purpureus (L.) Sweet. } \\
\text { (Lomba/kacang merah) }\end{array}$ & Thypus & 0.2 & Leaf & Oral & Single \\
\hline & $\begin{array}{l}\text { Senna alata (L.) Roxb. } \\
\text { (Galinggang/ketepeng) }\end{array}$ & Skin problem & 0.2 & Leaf & Oral, topical & Single \\
\hline \multirow[t]{4}{*}{ Lamiaceae } & $\begin{array}{l}\text { Hyptis capitata Jacq. (Talo- } \\
\text { talo/jukut pendul) }\end{array}$ & $\begin{array}{l}\text { High cholesterol, ear } \\
\text { problem, magical and } \\
\text { spiritual problem, } \\
\text { gastritis }\end{array}$ & 1.0 & $\begin{array}{l}\text { Leaf, } \\
\text { Stem }\end{array}$ & $\begin{array}{l}\text { Oral, } \\
\text { topical, } \\
\text { blown }\end{array}$ & $\begin{array}{l}\text { Mixture, } \\
\text { single }\end{array}$ \\
\hline & $\begin{array}{l}\text { Mentha piperita L. (Daun odol/leaf } \\
\text { mint) }\end{array}$ & $\begin{array}{l}\text { Dental and oral problem, } \\
\text { sore throat }\end{array}$ & 0.4 & Leaf & $\begin{array}{l}\text { Mouthwash, } \\
\text { oral }\end{array}$ & $\begin{array}{l}\text { Single, } \\
\text { mixture }\end{array}$ \\
\hline & $\begin{array}{l}\text { Ocimum basilicum L. (Kemangi } \\
\text { bombo) }\end{array}$ & Undefined ailments & 0.2 & Leaf & Oral & Single \\
\hline & $\begin{array}{l}\text { Plectranthus scutellarioides (L.) } \\
\text { R.Br. (Bulunangko/iler) }\end{array}$ & Cough & 0.2 & Leaf & Oral & Single \\
\hline Malvaceae & Sida acuta Burm.f. (Sidaguri) & Maternity care & 0.2 & Herba & Topical & Mixture \\
\hline Melastomaceae & $\begin{array}{l}\text { Melastoma malabathricum } \mathrm{L} . \\
\text { (Benduruk/harendong) }\end{array}$ & Smallpox & 0.2 & Leaf & Topical & Single \\
\hline Moraceae & $\begin{array}{l}\text { Ficus septica Burm.f. } \\
\text { (Lebanno/awar-awar) }\end{array}$ & Diabetic & 0.2 & Leaf & Oral & Single \\
\hline Musaceae & Musa paradisiaca L. (Pisang) & Maternity care & 0.2 & Stem & Topical & Mixture \\
\hline Myrtaceae & Psidium guajava L. (Jambu biji) & Diarrhea & 0.2 & Leaf & Oral & Single \\
\hline Phyllantaceae & $\begin{array}{l}\text { Sauropus androgynus (L.) Merr. } \\
\text { (Katu) }\end{array}$ & Breastmilk booster & 0.2 & Leaf & Oral & Single \\
\hline Piperaceae & $\begin{array}{l}\text { Piper umbellatum L. (Lepo- } \\
\text { lepo/obiungku) }\end{array}$ & $\begin{array}{l}\text { Undefined ailments, } \\
\text { hemorrhoids }\end{array}$ & 0.6 & Leaf & Oral, topical & Single \\
\hline Plantaginaceae & $\begin{array}{l}\text { Plantago major L. (Rumput } \\
\text { mangkok/leaf sendok) }\end{array}$ & Low back pain & 0.2 & Herba & Oral & Single \\
\hline \multirow[t]{7}{*}{ Poaceae } & $\begin{array}{l}\text { Bambusa vulgaris Schrad. } \\
\text { (Awo/bambu kecil) }\end{array}$ & Bone injury & 0.2 & Leaf & Topical & Mixture \\
\hline & $\begin{array}{l}\text { Dendrocalamus asper (Schult.) } \\
\text { Backer (Parring/bambu petung) }\end{array}$ & Bone injury & 0.2 & Root & Topical & Mixture \\
\hline & $\begin{array}{l}\text { Eleusine indica (L.) Gaertn. } \\
\text { (Kareje/rumput belulang) }\end{array}$ & Bone injury & 0.2 & Herba & Topical & Mixture \\
\hline & $\begin{array}{l}\text { Imperata cylindrica (L.) Rausch. } \\
\text { (Rea/alang-alang) }\end{array}$ & Bone injury, sore throat & 0.4 & $\begin{array}{l}\text { Root, } \\
\text { Shoot }\end{array}$ & Topical & Mixture \\
\hline & Oryza sativa L. (Padi) & Maternity care, fever & 0.4 & Fruit & Oral, topical & Mixture \\
\hline & $\begin{array}{l}\text { Saccharum spontaneum } \mathrm{L} . \\
\text { (Beang/Galagah) }\end{array}$ & Bone injury & 0.2 & Leaf & Topical & Mixture \\
\hline & $\begin{array}{l}\text { Themeda gigantea (Cav.) Hack. ex } \\
\text { Duthie (Tille) }\end{array}$ & Bone injury & 0.2 & Stem & Topical & Mixture \\
\hline \multirow[t]{2}{*}{ Rosaceae } & Fragaria vesca L. (Pajallak) & Undefined ailments & 0.2 & Leaf & Topical & Mixture \\
\hline & $\begin{array}{l}\text { Rubus fraxinifolius Poir. (Lelang } \\
\text { seba (rasberi) }\end{array}$ & Menstrual disorder & 0.2 & Fruit & Oral & Single \\
\hline Rubiaceae & Coffea arabica L. (Kopi) & High blood pressure & 0.2 & Leaf & Oral & Single \\
\hline \multirow[t]{4}{*}{ Solanaceae } & $\begin{array}{l}\text { Capsicum frutescens } \mathrm{L} . \\
\text { (Lombok/cabe) }\end{array}$ & Undefined ailments & 0.2 & Fruit & Topical & Single \\
\hline & $\begin{array}{l}\text { Solanum lycopersicum Lam. } \\
\text { (Tomat) }\end{array}$ & Heart disease & 0.2 & Fruit & Oral & Mixture \\
\hline & Solanum nigrum L. (Tananti/lauka) & $\begin{array}{l}\text { Open wound, skin problem, } \\
\text { breast cancer, diabetic }\end{array}$ & 0.8 & Leaf & Topical & Single \\
\hline & $\begin{array}{l}\text { Solanum torvum } \text { Sw. (Palolang } \\
\text { panggalak/terong pokak) }\end{array}$ & $\begin{array}{l}\text { High cholesterol, high } \\
\text { blood pressure, low back } \\
\text { pain }\end{array}$ & 0.6 & Leaf & Oral & Single \\
\hline Urticaceae & $\begin{array}{l}\text { Gonostegia hirta (Blume ex } \\
\text { Hassk.) Miq. (Karranbai) }\end{array}$ & Maternity care & 0.2 & Leaf & Topical & Mixture \\
\hline \multirow[t]{5}{*}{ Zingiberaceae } & $\begin{array}{l}\text { Alpinia galanga (L.) Willd. } \\
\text { (Allikku/lengkuas) }\end{array}$ & Skin problem & 0.2 & Rhizome & Topical & Single \\
\hline & Curcuma longa L. (Kunyit) & $\begin{array}{l}\text { Smallpox, dental and oral } \\
\text { problem, fever, } \\
\text { undefined ailments }\end{array}$ & 0.8 & Rhizome & Oral, topical & Mixture \\
\hline & $\begin{array}{l}\text { Curcuma zanthorrhiza Roxb. } \\
\text { (Paninik/kunyit) }\end{array}$ & Diarrhea & 0.2 & Rhizome & Oral & Single \\
\hline & $\begin{array}{l}\text { Zingiber montanum (J.Koenig) } \\
\text { Link ex A.Dietr. (Bangle) }\end{array}$ & Undefined ailments & 0.2 & Rhizome & Topical & Mixture \\
\hline & $\begin{array}{l}\text { Zingiber officinale Roscoe } \\
\text { (Leyya/jahe) }\end{array}$ & $\begin{array}{l}\text { Bone injury, cough, } \\
\text { headache, undefined } \\
\text { ailments }\end{array}$ & 1.0 & Rhizome & $\begin{array}{l}\text { Topical, } \\
\text { oral }\end{array}$ & Mixture \\
\hline
\end{tabular}


Traditional remedies are prepared before they are administered topically, orally or through any other routes. There were different modes of preparation, including boiling, soaking, squeezing, pounding, chewing, slicing, and roasting. The most widely followed method of preparation was boiling $(29.46 \%)$. The next two preferred modes were soaking and squeezing $(23.21 \%$ and $16.07 \%)$. There were $15.18 \%$ of the recorded remedies did not have any specific methods of preparation. Some preparations were run into two steps, heating on fire first followed by squeezing $(1.79 \%)$, or squeezing followed by boiling $(0.89 \%)$.

In the present study, various methods of usage of the medicinal preparations were recorded, including drinking, smearing, spouting, dripping, eating, sprinkling, applying as a paste, blowing, gargling, washing (hairs) and bathing. Drinking herbal preparation was the most frequent method of use $(33.93 \%)$. There were three types of smearing, only smearing (16.96\%), smearing and massaging (18.75\%), as well as smearing and drinking $(3.57 \%)$. Blowing, gargling, bathing and use as hair wash are the least used methods $(0.89 \%)$.

In the present study, 31 ailments were found to be treated by traditional medicinal remedies in the study area. Some ailments cannot be categorized into any specific categories (undefined). There were 9 usages of medicinal plant uses to treat such undefined ailments, as shown in Figure 7. The highest number of species and usages (9 species and 13 usages) were recorded for maternity care. The information of use shown in Figure 7 means that from 9 species to treat maternity care there are 13 usages of medicinal plants. Since one medicinal plant might have more than one part used with different methods of preparation.

The number of species used to treat certain diseases and the number of recorded information of use is the same, except in cases of maternity care, bone injury, magical and spiritual problems, open wound, gastritis, cough, fever, high cholesterol, neonatal care, and hemorrhoid. Eight ailments with least number of information of use and species (one information and species each) were menstrual disorder, anti-toxin, lice, headache, eye problem, appendicitis, thypus, and breast cancer.

\section{Discussion}

\section{Informant's profile}

As shown in Table 1, information of medicinal plants used to treat various ailments were obtained from five traditional healers. The fact that most of them are elderly shows that they still maintain their traditional knowledge. If they did not have a successor, the knowledge gap between the younger generation and their ancestors will be even greater (Musa et al. 2011; Amri and Kisangau 2012). Furthermore, none of the informants of this study have documented their knowledge, which is a threat to local knowledge. Low educational background of informants in Rongkong might be the reason why there was no written documentation. However, most of the traditional medicinal knowledge in Indonesia used to be transferred orally over generations and become community knowledge. Study on Sasak community found that most of the traditional medicine knowledge was transmitted verbally, only a few of them documented in babon (kind of book) tetamba/oat and lontar usada (Martini et al. 2017).

Earlier study on Tombulu community, Minahasa, North Sulawesi, has revealed that female traditional healers play an important role in improving public health as element of their culture (Rotty and Lumempouw 2013). This finding was similar to that of Rongkong Community. The number of female traditional healers was more than males which showed their important role in the community.

The informants thought that being a healer was not an occupation, but a social responsibility. They did not fix any tariffs and sometimes provide voluntary medical assistance to the community (Dermawan 2013). According to another study, people who seek advice from traditional healers commonly come from low-income families (Rotty and Lumempouw 2013). However in Rongkong, neither the rich nor the poor have any chance to choose, since their location is isolated and far from various health services. People tend to take the nearest available help when it comes to emergency situations.

\section{Medicinal plant diversity and use value (UV)}

Among 62 species of medicinal plants reported from Rongkong, 7 species belong to Poaceae. Six of them used by one informant to treat bone injury. Two of the six species in that remedy were bamboo (Dendrocalamus asper and Bambusa vulgaris). Previous research showed that another species of Bamboo, Bambusa arundinacea was used for lowering inflammation associated with bone fracture. Members of the Genus Bambusa contain benzoic acid, cyanogenic glycosides, silicon, calcium phosphorus and zinc which help heal fractures (Singh 2017). Another study found that the most common preparation of medicinal plants for treating bone fracture was applied externally as paste (Upadhya et al. 2012). One application of B.arundinaceae for fracture healing is by using its stem or leaf paste orally or topically (Singh 2017). The informant of this study used B. vulgaris and five other plants by soaking them in coconut oil, then the oil was applied and massaged on the injured part of the body.

Another medicinal species of Poaceae found in Rongkong was Eleusine indica. It was reported to have high calcium content that is needed in maintaining bone structure (Babu and Savithramma 2014). As revealed by interviews of this study, the informant has no information about the reason behind the Poaceae usage. There was no explanation by the informant for using Poaceae plants in his bone fracture formula. According to Traditional Chinese Medicine (TCM) theory, fracture pathology syndrome is characterized by pain, redness and swelling in the broken part, moreover, the stagnation of blood circulation slows down the healing process. Therefore, the function of the medicinal plant formula is to help reduce inflammation, accelerate blood circulation and enhance bone regeneration (Mukwaya et al. 2014). 
The second-largest number of species in this study belonged to Asteraceae. Members of this family were used to treat various ailments, two of them were used for treating gastritis. They were Ageratum conyzoides and Artemisia vulgaris. It has been reported that A.conyzoides has similar use in Makassar, South Sulawesi, and Mount Gede Pangrango, West Java (SMD et al. 2016, Tambaru 2017).

Five species belonging to Zingiberaceae are used as ingredients in the treatment formulas. They are Alpinia galanga, Curcuma longa, C. zanthorrhiza, Zingiber montanum and Z. officinale (Table 2). The rhizome of Zingiberaceae is popularly used to treat various ailments, including skin problems, fever, diarrhea and bone injury. There are 1,326 information of uses to treat various ailments using $C$. longa has been recorded from 644 traditional healers, and 281 information on C. zanthorrhiza from 172 traditional healers, from all over Indonesia (Subositi and Wahyono 2019). However, only two species of Curcuma were found to be used in Rongkong. Studies on distribution of Zingiberaceae at different altitudes in Semarang (Sari et al. 2012) revealed that at places more than $1250 \mathrm{~m}$ above sea level, only four species found, in which C.longa and Z.officinale are not mentioned.. Another report from southern slope of Mount Merapi, Yogyakarta has also found that three most widely used botanical families were Zingiberaceae, Asteraceae, and Lamiaceae (Nahdi and Kurniawan 2019), as found in the current study of Rongkong.

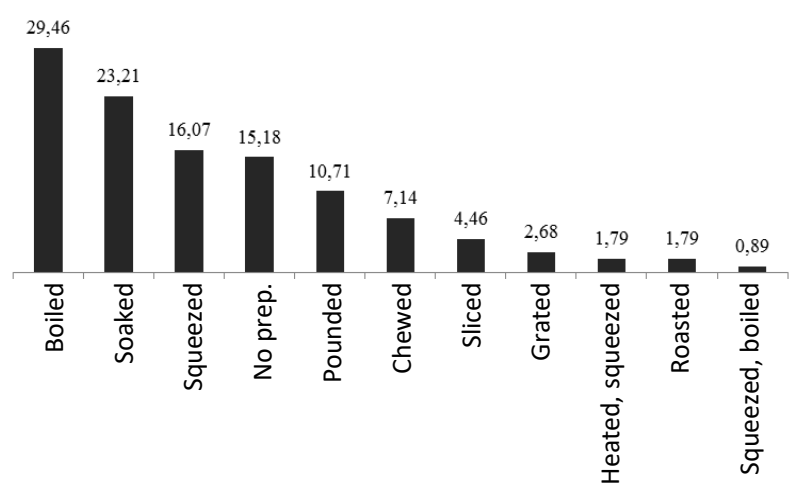

Figure 5. Percentage of different modes of preparation of traditional remedies

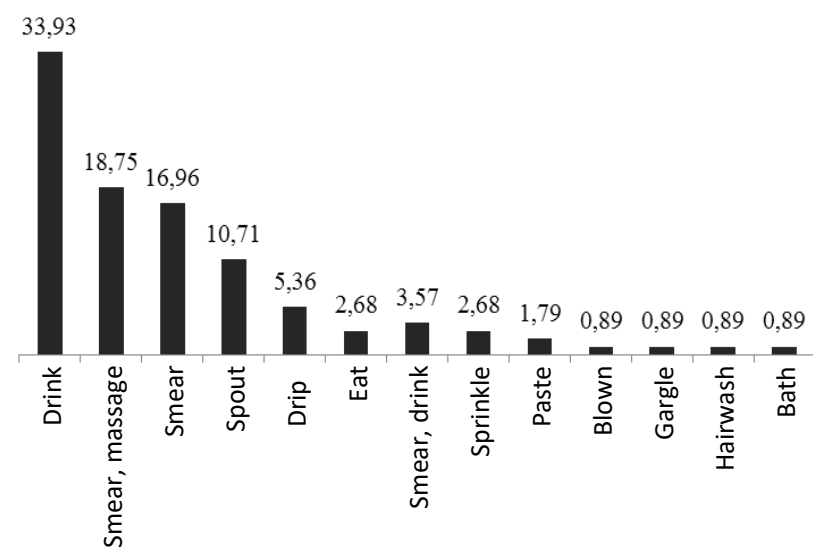

Figure 6. Different methods of usage of herbal preparations (\%)

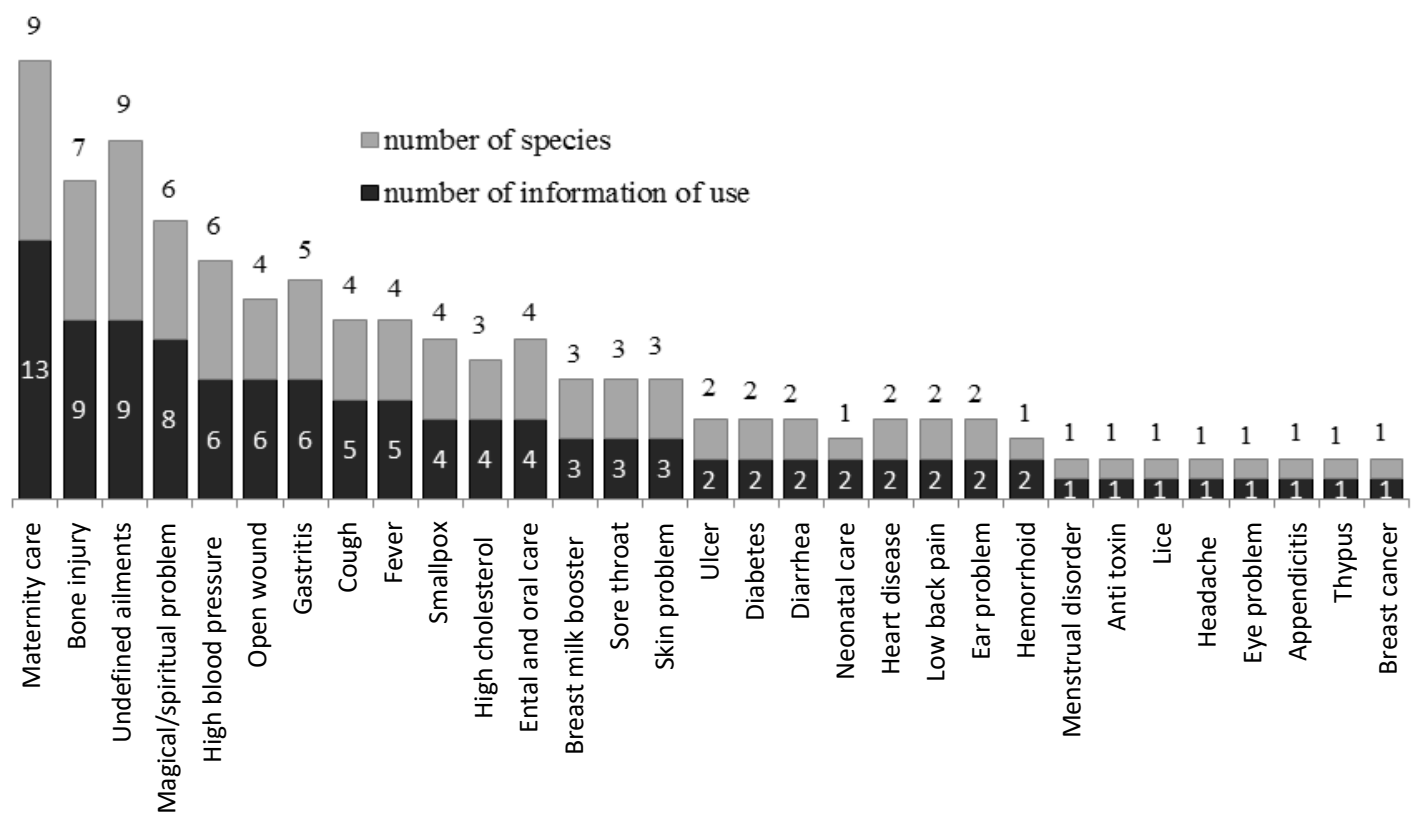

Figure 7. Number of medicinal plant species used and frequency of information against different ailments 
In Rongkong, A. cepa appears to be the most important medicinal species since it was cited in 11 remedies. The high use value describes that the relative importance of a species is more than other species, to the local community (Ullah et al. 2014). A. cepa used to treat measles, dental and oral problems, cough, ulcer, fever, in baby care and undefined ailments. Another study found that A. сера was widely used to treat various ailments in Asian countries, including digestive problems, skin diseases, metabolic disease, insect bite and others (Teshika et al. 2019). The wide distribution of certain species contributes to their high UV. Moreover, the awareness of local healers to use the species for health purposes affect the high level of UV (Ullah et al. 2014). A. cepa can grow better in the higher altitude, then in the lower land (Hilman et al. 2016). A. cepa probably has a wide distribution in Rongkong, since it is located at the altitude of 1,400 above sea level. Thus all informants 1 easily find and use this species as an ingredient of their remedy. A.conyzoides, H.capitata, and Z. Officinale were categorized as important species because each of them was locally used in five remedies. The number of ailments they could treat range between three and four, as shown in table 2.

Amaryllidaceae has the highest FUV among all families (1.20) which implies that it is an important family of medicinal plants, in the current study. This family consists of only two medicinal species, and one of them has the highest UV. On the contrary, Poaceae has the largest number of species, but its FUV was low. It is because of 7 medicinal species of Poaceae has only one use report per species.

\section{Parts used, modes of preparation and methods of use}

More than $50 \%$ of the information recorded in this study used leaf as the medicinal ingredient. Leaf is widely used in herbal remedies since it is available in abundant amount and easily harvested. Furthermore, leaves accumulate chemical contents that possess medicinal properties. The use of leaves is also in line with the principle of conservation, as leaf harvesting does not damage or cause death of the plants. Instead, if the use of roots and stems are more dominant, it can threaten the preservation of a particular plant (Amri and Kisangau 2012; Fiscal 2017; Supiandi et al. 2019). Many communities in other areas also use leaves as the main ingredient in their traditional medicine (Giday et al. 2010; Nulfitriani et al. 2015; Silalahi et al. 2018). In this present study, informants had no specific consideration in selecting the proper part of the plant to treat each ailment, they did it based on traditional knowledge. It is to be noted that the local community still lack knowledge about the most useful part of the plant, as well as the precise time of harvesting (Alzweiri et al. 2011).

There were various modes of drug preparation recorded in this study, including boiling, chewing, grating, heating, squeezing, pounding, roasting, slicing, and soaking. The first three most frequently used modes were boiling, soaking and squeezing. People usually use water to boil the ingredients. Similar to an earlier study from Jordan
(Alzweiri et al. 2011), water was the most common solvent added to traditional remedies of Rongkong. The next common solvents were coconut oil as well as palm oil. In specific cases, the healers use honey and coconut water to dissolve the herbal ingredients.

Ullah et al. (2014) categorized routes of administration of traditional remedies into topical, oral, eardrop, necklace, pulmonary, brush and hair oil. In this present study, the recorded routes are oral, topical, ear drop, eye drop, blown hair wash, mouthwash, and bath. Topical is an important route to treat many ailments with various methods of application, including smearing, as a paste, spouting, and sprinkling, while oral is by drinking and eating. Smearing was the most dominant method of use in topical group. It is divided into two methods, the first was only smearing for problems like ulcer, open wound, fever and as breast milk booster, and the second was smearing followed by massaging on the affected part of the body. Similar methods were also found in previous ethnomedicinal studies (Nawangningrum et al. 2010; Rotty and Lumempouw 2013; Sutrisna et al. 2019).

The method of using herbal medicine by chewing and spouting by the healers are common in Indonesia's traditional medicinal practice. Earlier study in Barus Jahe, North Sumatra reported that traditional medicinal practice systems consist of three methods, herbal formula, massage and spouting (Sembiring 2015). Some remedies for smallpox in Bali consists of spouting herbal drug into the affected parts of the body. These treatments were stated in Lontar Usada, an ancient traditional medicinal document of Bali (Sutrisna et al. 2019).

\section{Medicinal plants used to treat ailments}

As shown in Figure 5, there was 13 information on medicinal plants used in the treatment of childbirth. Medicinal plants are rarely used at the early stage of pregnancy, pregnants in Rongkong use it at the end stage of pregnancy, to accelerate childbirth and adjust the fetus position. At delivery and postpartum phase, they use it to remove the placenta and relieve postpartum pain. The use of traditional medicine was common during postpartum recovery, especially given by informants who played role as traditional midwives. They usually prepare traditional medicine for women and children, like for promoting breastfeeding and baby care. The use of medicinal plants by pregnant women at different stages of pregnancy is common in other countries as well (Ali-Shtayeh et al. 2015). Previous study in Kry Ethnic Group, Lao PDR documented the use of 15 species of medicinal plants for postpartum treatment. People avoided the use of traditional medicine during pregnancy, however when the baby is born, both mother and her baby start to use it. They use it to reduce postpartum pain and relieve health problems of the babies (Lamxay et al. 2011).

Undefined ailments were health problems that can not be categorized into certain types of diseases. The informants can clearly state the symptoms, however, the information did not lead to a specific category of disease medically. Some examples are (i) Redness in breast, 
without lumps and no breastfeeding (2 information), (ii) Swelling and redness on the toes (1 information), and (iii) Pain in the stomach, vomiting, headache, difficulty in breathing and fever (6 information). The informants mention the third one as cika disease, cured with cika remedy which consists of Allium cepa, Acorus calamus, Curcuma longa, Zingiber officinale, Z. montanum, and Fragaria vesca. All the ingredients were chewed up and then spout directly from the mouth into painful parts of the body.

There is 8 information about medicinal plants used to heal bone injury, applied by an informant who has specialization in treating bone fractures. Rotty and Lumempouw (2013) reported that Tombulu Community in Minahasa, North Sulawesi divided illness into 2 types, physical and non-physical illness. Physical illness is a disease, the cause of which can be clearly identified and treatment is available, but not for non-physical. The reason is interference from spirits or the influence of a person's magical arts. Two informants in this present study believe in diseases that arise due to magical or spiritual influences. Based on the interview, this kind of disease was characterized by fever, the body feels cycle of hot and cold. Both informants had different ingredients of remedies, but the method of use was similar, by splashing on the patient's face. These methods were common in traditional medication among Indonesian healer. There are six information about plants used to treat this ailment, those were Ageratum conyzoides, Bidens pilosa, Drymaria cordata, Commelina bengalensis, Jatropha curcas, and Hyptis capitata. A.conyzoides and H.capitata were used by both informants. In Assam, India, flowers of $A$. conyzoides used to make kind of rice beer which is used during the ritual in dispelling bad spirits (Sharma and Pegu 2011). There are certain ethnicities that believe that some diseases arise because of someone's bad attitude or evil spirit influence (Dwivedi and Dwivedi 2015; Oknarida et al. 2019).

Rongkong community still relies on traditional healers since the location of this sub-district is isolated and far from public health services. Traditional healer is the nearest help that is expected to treat the community's health problems. Traditional healers have managed both the natural resources and traditional healthcare system over generations. However, effective successors and proper documentation are decisively needed to fulfill the community's needs in the future. Both traditional knowledge and medicinal plant diversity possessed by Rongkong community are valuable local assets that must be maintained and developed for greater benefits.

\section{ACKNOWLEDGEMENTS}

We would like to acknowledge the Rongkong Traditional Healers and Rongkong Public Health Center for all the valuable information that they have shared. We would also like to thank Medicinal Plant and Traditional Medicine Research and Development Centre for funding the study and all team members of Research on
Ethnomedicine Knowledge and Medicinal Plant-Based on Community in Indonesia (RISTOJA) 2017, including technical, taxonomist and data management team. We are grateful to Dr. Yuli Widiyastuti, and Dr. Sari Haryanti, for the writing assistance, and also Tri Wijayanti, Andi M. Yusuf and M. Riswan Ramli (Rongkong Data Collection Team) for all efforts in collecting the data and medicinal plant specimen.

\section{REFERENCES}

Ali-Shtayeh MS, Jamous RM, Jamous RM. 2015. Plants used during pregnancy, childbirth, postpartum and infant healthcare in Palestine. Compl Ther Clin Pract 21: 84-93. DOI: 10.1016/j.ctcp.2015.03.004

Alzweiri M, Sarhan AA, Mansi K, Hudaib M, Aburjai T. 2011. Ethnopharmacological survey of medicinal herbs in Jordan, the Northern Badia region. J Ethnopharmacol 137: 27-35. DOI: 10.1016/j.jep.2011.02.007

Amri E, Kisangau DP. 2012. Ethnomedicinal study of plants used in villages around Kimboza forest reserve in Morogoro, Tanzania. J Ethnobiol Ethnomed 8: 1-9. DOI: 10.1186/1746-4269-8-1

Babu RH, Savithramma N. 2014. Studies on mineral analysis of grasses of Poaceae. Intl J Pharm Sci 4: 526-531.

Cheikhyoussef A, Shapi M, Matengu K, Mu Ashekele H. 2011. Ethnobotanical study of indigenous knowledge on medicinal plant use by traditional healers in Oshikoto region, Namibia. J Ethnobiol Ethnomed 7: 10. DOI: 10.1186/1746-4269-7-10

Dermawan R. 2013. Peran Battra dalam Pengobatan Tradisional pada Komunitas Dayak Agabag di Kecamatan Lumbis Kabupaten Nunukan. Sosiologi Konsentrasi 1: 50-61. [Indonesian]

Dwivedi SDS, Dwivedi A. 2015. Herbal Remedies for Respiratory Diseases among the Natives of Madhya Pradesh, India. Am J Life Sci Res 3 (2): 158-162.

Fiscal R. 2017. Ethnomedicinal Plants Used by Traditional Healers in Laguna, Philippines. Asia Pac J Multidisciplinary Res 5: 132-137.

Gazzaneo LRS, de Lucena RFP, de Albuquerque UP. 2005. Knowledge and use of medicinal plants by local specialists in a region of Atlantic Forest in the state of Pernambuco (Northeastern Brazil). J Ethnobiol Ethnomed 1: 9. DOI: 10.1186/1746-4269-1-9

Ghimire K, Adhikari M, Uprety Y, Chaudhary R. 2018. Ethnomedicinal Use of Plants by the Highland Communities of Kailash Sacred Landscape, Far-west Nepal. Acad J Med Plants 6 (11): 365-378. DOI: 10.15413/ajmp.2018.0171.

Giday M, Asfaw Z, Woldu Z. 2010. Ethnomedicinal study of plants used by Sheko ethnic group of Ethiopia. J Ethnopharmacol 132: 75-85. DOI: 10.1016/j.jep.2010.07.046

Hilman Y, Rosliani R, Palupi ER. 2016. Pengaruh Ketinggian Tempat Terhadap Pembungaan, Produksi, dan Mutu Benih Botani Bawang Merah. Jurnal Hortikultura 24: 154-161. DOI: 10.21082/jhort.v24n2.2014.p154-161. [Indonesian]

Hoffman B, Gallaher T. 2007. Importance Indices in Ethnobotany. Ethnobot Res Appl 5: 201. DOI: 10.17348/era.5.0.201-218

Lamxay V, de Boer HJ, Björk L. 2011. Traditions and plant use during pregnancy, childbirth and postpartum recovery by the Kry ethnic group in Lao PDR. J Ethnobiol Ethnomed 7: 14. DOI: 10.1186/17464269-7-14

Martini D, Haq H, Sutrisno B. 2017. Perlindungan Hukum Terhadap Pengetahuan Obat-Obatan Tradisional Dalam Rezim Hak Kekayaan Intelektual (HKI) Indonesia (Studi Pada Masyarakat Tradisional Sasak). Jurnal Hukum dan Peradilan 6: 67-90. DOI: 10.25216/JHP.6.1.2017.67-90. [Indonesian]

Mukwaya E, Xu F, Wong M-S, Zhang Y. 2014. Chinese herbal medicine for bone health. Pharm Biol 52: 1223-1228. DOI: 10.3109/13880209.2014.884606.

Musa MS, Abdelrasool FE, Elsheikh EA, Mahmoud ALE, Yagi SM. 2011. Ethnobotanical study of medicinal plants in the Blue Nile State, South-eastern Sudan. J Med Plants Res 5: 4287-4297.

Nahdi MS, Kurniawan AP. 2019. The diversity and ethnobotanical study of medicinal plants in the southern slope of Mount Merapi, Yogyakarta, Indonesia. Biodiversitas 20: 2279-2287. DOI: $10.13057 /$ biodiv/d200824 
Nawangningrum D, Widodo S, Suparta IM, Holil M. 2010. Kajian Terhadap Naskah Kuna Nusantara Koleksi Fakultas Ilmu Pengetahuan Budaya Universitas Indonesia: Penyakit Dan Pengobatan Ramuan Tradisional. Hubs-Asia 10. Available from: http://hubsasia.ui.ac.id/old/index.php/hubsasia/article/view/86 (March 27, 2019).

Nulfitriani N, Ramadhanil R, Yuniati E. 2015. Pemanfaatan Tumbuhan Sebagai Obat Tradisional Pada Suku Tolitoli di Desa Pinjan Sulawesi Tengah. Biocelebes 7. DOI: 10.22487/j25805991.2013.v7.i2.3925 [Indonesian]

Oknarida S, Husain F, Wicaksono H. 2019. Kajian Etnomedisin Dan Pemanfaatan Tumbuhan Obat Oleh Penyembuh Lokal Pada Masyarakat Desa Colo Kecamatan Dawe Kabupaten Kudus. Solidarity: J Educ Soc Culture 7 (2): 480-500. [Indonesian]

Rotty TA, Lumempouw F. 2013. Makna Tokoh Penyembuh Masyarakat Tombulu. Duta Budaya, Jurnal Fakultas Sastra Universitas Sam Ratulangi 2013: 91-102. [Indonesian]

Sari HM, Utami S, Wiryani E, Murningsih M, Perwati LK. 2012. Distribusi Famili Zingiberaceae pada ketinggian yang berbeda di Kabupaten Semarang. Bioma: Berkala Ilmiah Biologi 14: 1-6. DOI 10.14710/bioma.14.1.1-6 [Indonesian]

Sembiring S. 2015. Pengetahuan dan pemanfaatan metode pengobatan tradisional pada masyarakat Desa Suka Nalu Kecamatan Barus Jahe. Perspektif Sosiologi 3: 104-117. [Indonesian]

Sharma UK, Pegu S. 2011. Ethnobotany of religious and supernatura beliefs of the Mising tribes of Assam with special reference to the "Dobur Uie." J Ethnobiol Ethnomed 7: 16. DOI: 10.1186/1746-42697-16

Silalahi M, Nisyawati N, Walujo E, A. Mustaqim W. 2018. Etnomedisin Tumbuhan Obat oleh Subetnis Batak Phakpak di Desa Surung Mersada, Kabupaten Phakpak Bharat, Sumatera Utara. Jurnal Ilmu Dasar 19: 77-92. [Indonesian]

Singh V. 2017. Medicinal plants and bone healing. Nat J Maxillofacial Surg 8: 4-11. DOI: 10.4103/0975-5950.208972

SMD R, Rostiana O, Pribadi ER, Hernani H. 2016. Penggalian Iptek Etnomedisin di Gunung Gede Pangrango. Buletin Penelitian Tanaman Rempah dan Obat 18: 13-28. DOI: 10.21082/bullittro.v18n1.2007.\%p [Indonesian]
Subositi D, Wahyono S. 2019. Study of the genus Curcuma in Indonesia used as traditional herbal medicines. Biodiversitas J Biol Divers 20. DOI: $10.13057 /$ biodiv/d200527

Suharmiati S, Handayani L, Kristiana L. 2012. Faktor-faktor yang Memengaruhi Keterjangkauan Pelayanan Kesehatan di Puskesmas Daerah Terpencil Perbatasan di Kabupaten Sambas (Studi Kasus di Puskesmas Sajingan Besar). Buletin Penelitian Sistem Kesehatan 15 (3): 223-231. [Indonesian]

Supiandi MI, Mahanal S, Zubaidah S, Julung H, Ege B. 2019. Ethnobotany of traditional medicinal plants used by Dayak Desa Community in Sintang, West Kalimantan, Indonesia. Biodiversitas 20 (5): 1264-1270. DOI: 10.13057/biodiv/d200516

Sutrisna INGT, Widyastuti NLG, Cahyadi KD. 2019. Kajian Pengobatan Tradisional Cacar Menurut Terjemahan Lontar Usada Kacacar. Jurnal Riset Kefarmasian Indonesia 1: 41-55. DOI: 10.33759/jrki.v1i1.12 [Indonesian]

Tambaru E. 2017. Keragaman Jenis Tumbuhan Obat Indigenous Di Sulawesi Selatan. Jurnal Ilmu Alam dan Lingkungan 8: 7-13. [Indonesian]

Teshika JD, Zakariyyah AM, Zaynab T, Zengin G, Rengasamy KR, Pandian SK, Fawzi MM. 2019. Traditional and modern uses of onion bulb (Allium cepa L.): a systematic review. Crit Rev Food Sci Nutr 59: S39-S70. DOI: 10.1080/10408398.2018.1499074

Ullah S, Rashid Khan M, Ali Shah N, Afzal Shah S, Majid M, Asad Farooq M. 2014. Ethnomedicinal plant use value in the Lakki Marwat District of Pakistan. J Ethnopharmacol 158: 412-422. DOI: 10.1016/j.jep.2014.09.048

Upadhya V, Hegde HV, Bhat S, Hurkadale PJ, Kholkute SD, Hegde GR. 2012. Ethnomedicinal plants used to treat bone fractures from NorthCentral Western Ghats of India. J Ethnopharmacol 142: 557-562. DOI: 10.1016/j.jep.2012.05.051

Wartika Y, Yuniati E, Ramadhanil R. 2015. Kajian Etnobotani pada Masyarakat Adat Rongkong di Desa Rinding Allo Kecamatan Limbong Kabupaten Luwu Utara Sulawesi Selatan. Biocelebes 7. DOI: 10.22487/j25805991.2013.v7.i1.3903. [Indonesian]

Weckerle CS, de Boer HJ, Puri RK, van Andel T, Bussmann RW, Leonti M. 2018. Recommended standards for conducting and reporting ethnopharmacological field studies. J Ethnopharmacol 210: 125-132. DOI: 10.1016/j.jep.2017.08.018 